\title{
Patient Safety in the Operating Room
}

\author{
Warren A. Ellsworth IV, M.D. ${ }^{1}$ and Ronald E. Iverson, M.D. ${ }^{2}$
}

Maintaining patient safety in the operating room is a major concern of surgeons, hospitals, and surgical facilities. Circumventing preventable complications is essential, and the pressure to avoid these complications during elective cosmetic surgery is especially important. Traditionally, nursing and anesthesia staff have managed patient positioning and most safety issues in the operating room. As the number of office-based procedures in the plastic surgeon's practice increases, understanding and implementation of patient safety guidelines by the plastic surgeon is of increasing importance.

Key aspects of patient safety in the operating room include thoughtful patient positioning, ocular protection, proper handling of electrocautery, and airway management. If performed correctly with attention to certain anatomic landmarks, preoperative positioning of the patient can prevent nerve injury and postoperative joint or muscle pain. In this article we discuss proper patient positioning with attention to protection against nerve palsy. Further, we discuss common patient positions on the operative table and highlight special concerns associated with each position. Other safety issues including prevention of ocular injury and proper management of electrocautery are discussed.

Responsibility of postoperative complications ultimately lies with the surgeon. Careful attention to patient safety guidelines is of paramount importance to surgeons, especially during elective cosmetic procedures. Attention to detail in patient positioning, eye protection, and bovie use can help avoid unnecessary perioperative complications and significantly improve the patient's cosmetic surgery experience.

KEYWORDS: Patient safety, operating room, patient positioning, ocular injury, electrosurgery, nerve injury

In the landmark publication To Err Is Human, the Institute of Medicine explains that between 44,000 and 98,000 Americans die each year because of medical error. ${ }^{1}$ In this report, the authors highlight patient safety as a key area in need of improvement in hospitals today. Patient safety in the operating room is a major concern for physicians, hospitals, and surgical facilities alike. Physicians have an obvious investment in the safety of their patients, and surgeons are ultimately responsible for all activity that occurs during a surgical procedure. Hospitals and surgical facilities are reviewed by many national organizations that specifically review patient safety issues when determining accreditation status. Further, the malpractice crisis in our country only heightens the level of attention placed on safety issues in the operating room.

Maintaining patient safety during cosmetic procedures is especially important because of the elective nature of the operation. A complication of nerve palsy due to poor positioning or electrocautery burn due to a misplaced Bovie pad can be devastating to both surgeon and patient. In the hospital setting, much of
${ }^{1}$ Division of Plastic Surgery, Baylor College of Medicine, Houston, Texas; ${ }^{2}$ The Plastic Surgery Center, Stanford University School of Medicine, Pleasanton, California.

Address for correspondence and reprint requests: Warren A. Ellsworth IV, M.D., Division of Plastic Surgery, Baylor College of Medicine, 1709 Dryden, Suite 1600, BCM Mail Stop: 630, Houston, TX 77030 .
Optimization of Patient Safety in Cosmetic Surgery; Guest Editors, C. Bob Basu, M.D., M.P.H. and Gustavo A. Colon, M.D.

Semin Plast Surg 2006;20:214-218. Copyright (C) 2006 by Thieme Medical Publishers, Inc., 333 Seventh Avenue, New York, NY 10001, USA. Tel: +1(212) 584-4662.

DOI 10.1055/s-2006-951578. ISSN 1535-2188. 
the positioning and patient preparation is completed by nurses and anesthesiology staff. Now that plastic surgeons are performing more cosmetic procedures in the office-based surgical setting, the importance of understanding proper safety guidelines and preoperative management of patient positioning becomes an important issue. In this article, we discuss several key aspects of patient safety in the operating room. Basic considerations that surgeons should revisit before each procedure are attention to patient positioning, proper handling of electrocautery, corneal protection, and airway management. Attention to these issues preoperatively may prevent complications that can become significant problems in the postoperative period.

\section{PATIENT POSITIONING}

Positioning the patient before the start of an operation is a fundamental element in a successful procedure. Poor patient positioning can result in a more challenging procedure and can produce less satisfying results. Both anesthesiologist and surgeon should agree upon the patient's position preoperatively. In doing so, the team should always take into account that anesthetized patients cannot make the surgeon aware of compromised positions. Further, the team must consider the patient's medical comorbidities and their limitations on positioning. For example, patients with severe osteoporosis are predisposed to bone fractures even after a minimal insult such as rolling from the operating room table to the bed. $^{2}$ Also, obese patients are more difficult to position because their extremities cannot move to the same degree as in slender people and, if stretched, nerve injury results more easily.

Patients who undergo a procedure that requires a long operative time are at risk for pressure necrosis of skin and underlying tissue. Studies have shown that a pressure of just $70 \mathrm{~mm} \mathrm{Hg}$ applied for a period of 2 hours or longer can result in irreversible tissue ischemia. ${ }^{2}$ Areas especially susceptible include the skin over the forehead, iliac crests, and bony prominences on the arms and legs. ${ }^{2}$ Foam "eggcrates" or similar foam sponges placed over these areas and over the heels in the supine patient can help decrease the risk of injury. Also, periodic elevation of the area during the procedure allows a greater amount of blood flow.

Peripheral nerve injury is usually a preventable complication of poor patient positioning. Nerves are injured by one of two mechanisms: stretch or compression. $^{2}$ If the nerve is pulled between two fixed points, stretch injury occurs. Compression injury is largely due to loss of protective muscle tone and pressure between two fixed points. The final result is the same: nerve ischemia related to poor blood flow. ${ }^{3}$ Whereas awake patients usually feel the effects of nerve ischemia and adjust body position, the anesthetized patient is unable to sense or respond to such signs.

There are three degrees of nerve injury. The first is neuropraxis, which carries the best prognosis. Neuropraxis is a response to compression with only slight evidence of demyelination and no axonal degeneration. Full recovery is expected within 6 weeks without residual function loss. ${ }^{3}$ The second level of nerve injury is axonotmesis, which occurs when there is destruction of the axons within a nerve sheath that remains intact. ${ }^{2}$ The distal axons degenerate and the proximal axons use the intact nerve sheath to guide regeneration at a rate of $1 \mathrm{~mm}$ per day. Function is initially lost but can return over a period of months to a year, depending on the length of the nerve. ${ }^{3}$ The final and most severe nerve injury is neurotmesis, which results in disruption of axon, sheath, and connective tissue covering. Degeneration of the distal axon occurs, but nerve recovery and regeneration are less likely. ${ }^{3}$ Painful neuromas can develop because of disorganized axonal regeneration. Surgical intervention with resection of nerve endings and reapproximation may help restore function.

In a study by the American Society of Anesthesiologists, nerve injury accounted for $15 \%$ of legal claims, and the most commonly injured nerves include the brachial plexus, ulnar nerve, and radial nerve. ${ }^{3,4}$ These injuries are usually not permanent, but even temporary loss of the brachial plexus is an unacceptable outcome for some patients after an elective cosmetic procedure.

\section{The Brachial Plexus and Its Branches}

Brachial plexus injury is a well-documented complication of long procedures when arms are at right angles to the body or hyperextended. A group of plastic surgeons reported a case of an obese woman who underwent bilateral reduction mammoplasty, abdominoplasty, and liposuction with postoperative brachial plexus denervation bilaterally. ${ }^{5}$ The patient slowly regained motor and sensory function over several months. The brachial plexus injury is due to stretch between the coracoid process and head of the humerus. This stretch is seen at 90 degrees of arm abduction and is worsened as the arm becomes more hyperextended. ${ }^{2}$ Plastic surgeons should be aware of this complication, as it is quite common in breast surgery to keep a patient's arms in the abducted position. Surgeons should be especially concerned in obese patients because the tension applied to the plexus can be larger at a lesser degree of abduction because of the large arm circumference. ${ }^{5}$ In these patients, abduction should be less than 80 degrees to prevent injury. Further, flexion of the head with downward displacement of the shoulder can put undue stress on the brachial plexus and should be avoided.

The ulnar and radial nerves are branches of the brachial plexus that can be injured if inappropriately 
positioned. The cubital tunnel compression syndrome occurs because the ulnar nerve lies superficial in the tunnel at the elbow. Some have postulated that the syndrome occurs when the arm is fixed at the patient's side or abducted with the forearm pronated. In these positions, the cubital tunnel is placed in contact with the table surface and is subject to compression injury. Supination allows the olecranon process to protect the nerve from contact with the flat surface, possible reducing injury. ${ }^{3}$ Also, flexion of the elbow more than 90 degrees causes a decrease in the cubital tunnel size by more than $90 \%$, possibly compressing the nerve further. ${ }^{3}$ To best avoid ulnar nerve injury, surgeons should confirm appropriate padding and avoid the preceding arm positions.

Like the ulnar nerve, the radial nerve lies superficial as it wraps around the proximal third of the humerus in the radial groove. Palsy of this nerve is less common than that of the ulnar, but improper padding of the arm along the arm board can predispose to compression injury and temporary functional loss. Current recommendations suggest protecting this area of the arm with soft padding and avoiding circumferential tape in the area of the proximal humerus. Studies have shown, however, that even with appropriate positioning and padding some patients still suffer from nerve palsy. ${ }^{3,4}$ Discussing the possibility of temporary nerve palsy preoperatively may avoid confusion or anger during the postoperative period if the complication occurs.

\section{Surgical Positions}

A large spectrum of positions is used in the operating room. In cosmetic surgery, however, the most commonly used positions include supine, prone, and sitting. Supine is the most common position and allows the surgeon adequate access to the face, breast, abdomen, and anterior aspects of the arms, legs, and thighs. Because there is no pressure gradient above or below the heart, the physiologic consequences for the cardiovascular system are minimal. ${ }^{2}$ With the patient in the supine position, the surgeon should be aware of the head and cervical spine and ensure that these are midline. Sudden movements of the head can place undue stress on the brachial plexu, causing stretch injury. There are even reports of vertebral artery dissection after vigorous movement of the head and neck during surgery. ${ }^{6}$ Other guidelines include protection of the heels, elbows, and upper arms with soft padding. When the arms are abducted, they should be at an angle of less than 90 degrees with elbows slightly flexed and hands in the supine position. This offers the greatest protection from palsy of the branches of the brachial plexus. ${ }^{3,4,6}$

Plastic surgeons utilize the prone position more commonly than ever before. In reconstructive surgery it offers obvious access to the sacrum for pressure ulcers as well as to the gluteus and latissimus muscles for use in reconstructive efforts. In cosmetic surgery this position is useful for liposuction, circumferential body lifts, thigh lifts, and buttock surgery. The prone position is usually achieved by intubation on the patient's stretcher and movement after anesthesia onto the patient's abdomen. Care must be given to avoid excessive pressure on the eyes, ears, nose, breasts, and male genitalia. ${ }^{2}$ Basic padding requirements include using a gel shoulder roll under the upper chest as well as one supporting the pelvis. This position allows space for breasts, abdomen, and genitalia to rest free of pressure. Pillows or foam eggcrates should be placed under the knees and ankles to avoid pressure on these bony prominences.

Special attention must be given to the face and head when using the prone position. There have been accounts of vertebral artery dissection, thrombus, and stroke after a plastic surgery procedure in the prone position. ${ }^{7}$ This devastating complication occurred several days after an uneventful procedure in which the patient's head was laid on its side on a pillow to avoid pressure on the breathing tube. The position caused obstruction of the vertebral artery, dissection with thrombus formation, and eventual embolus causing stroke. To avoid this serious complication, surgeons should use standard foam prone pillows that allow stabilization of the neck in the neutral position and direction of the endotracheal tube away from the face to avoid excessive pressure. ${ }^{7}$ Finally, attention should also be given to the arm position to avoid stretch or compression injury to the brachial plexus or its branches. Eggcrate padding of the arm on standard arm boards with abduction of both the shoulder and elbow at less than 90 degrees allows maximal protection of the nerves. ${ }^{2,3,7}$ Following these basic guidelines for the prone position allows plastic surgeons to avoid these neural and vascular complications.

Less commonly used positions in cosmetic surgery include the lithotomy, lateral decubitus, and seated positions. Although each position has its own risks and benefits, the approach outlined previously for protection of the head, neck, and extremities from damage to the nerve and vascular structures maximizes safety in any position.

\section{OCULAR INJURY}

Eye injury after general anesthesia is infrequent but can result in serious complications such as visual impairment. Roth et $\mathrm{al}^{8}$ retrospectively studied a group of 60,000 patients over a 4-year period and found the rate of ocular injury to be $\sim 0.06 \%{ }^{8}$ The most common type of injury is corneal abrasion, and others include conjunctivitis, chemical injury, and blindness. ${ }^{8}$ 
The cornea is easily abraded because of reduced lacrimation during anesthesia. ${ }^{2}$ It is at increased risk for injury if face masks are improperly applied or if surgical drapes are manipulated while the eyes remain open. ${ }^{8}$ For these reasons, the eyelids should be closed and held closed with tape prior to any movement after intubation. Cosmetic surgery of the face limits the ability to protect the eyes in this way. There is little in the literature regarding the most appropriate way to protect the eyes during face or eye surgery, but plastic corneal protectors coated with lubricant and inserted under the eyelids seem to be an effective and safe method. Many surgeons, however, are concerned that corneal protectors distort eyelid anatomy and therefore do not use them during blepharoplasty or face lift. At a minimum, eye lubricant should be used if the eyelids cannot be taped or protected otherwise. This simple intervention may help avoid corneal abrasion with suture or, worse, globe trauma.

In some positions, such as prone, a significant amount of pressure can be applied to the eyes. If the pressure on the eye is greater than venous pressure, blood outflow is impeded and retinal hemorrhage is possible. If the pressure on the eye is greater than arterial pressure, inflow of oxygenated blood is stopped and retinal ischemia and blindness ensue. ${ }^{3}$ Although loss of vision is rare, this complication is devastating and should be avoided at all costs. There are no data to prove its benefit, but many plastic surgeons currently use goggles (Dupaco, Oceanside, CA) and prone pillows (Orthopedic Systems, Union City, CA) to protect the eyes and face from undue pressure in the prone position. ${ }^{7}$

\section{ELECTROSURGERY}

Electrosurgery describes the use of the Bovie or electrocautery. In electrosurgery, an electrode is used to create a circuit and conduct heat into a concentrated area. The electrosurgical generator is the source of electrons and voltage. ${ }^{2}$ The circuit is composed of the generator, the electrode, the patient, and the patient return electrode. ${ }^{9}$ The electrons overcome the impedance of the patient's tissue, creating the heat produced by the electrode pen. ${ }^{2}$ In monopolar electrosurgery, the return electrode is the grounding pad placed elsewhere on the patient's body. In bipolar electrosurgery, the return electrode is the second prong of the pickups, thereby concentrating the current at the area of interest and avoiding current through the patient's body. ${ }^{9}$

Over the years, electrosurgery has evolved. At one time, the circuit to the ground was completed by the path of least resistance on the patient. This caused current division and allowed alternate site burns at areas of least resistance even when grounding pads were in place. Today, however, generators come equipped with isolated generators that complete the circuit within the unit themselves. These isolated generators eliminated alternate site burns but did nothing to protect from return electrode burns at the site of grounding pad placement. ${ }^{9}$ In fact, between 1996 and 1998, 628 cases of grounding pad burns were documented by the Food and Drug Administration. ${ }^{10}$

The only difference between the active electrode, or the Bovie tip, and the grounding electrode is size and conductivity. ${ }^{2,9}$ The high current of the electrode tip is dissipated at the grounding pad over a larger surface area, eliminating the power of the current to burn the patient at the return site. If there is poor contact between the grounding pad and the patient, however, severe burns can develop as the current becomes concentrated on a smaller surface area. Inadvertent movement of the pad or poor placement can cause severe burns in a patient undergoing a simple procedure. To avoid this devastating complication, the grounding pads should be placed in areas without excessive hair, bony prominences, or adhesive failure and should be applied in such a way as to guarantee that the entire gel pad is in contact with the patient. ${ }^{2,9}$

Burns can also occur as a result of inadvertent placement of an active electrode on the patient's body. This can be avoided with meticulous placement of the tip of the instrument when it is not in use. For example, the tip should never be left on the patient's body because accidental activation will cause a burn injury. By following these simple guidelines and paying attention to the active electrode as well as appropriate placement and monitoring of the grounding pad, unintentional burns can virtually be eliminated.

\section{ENDOTRACHEAL ABRASION INJURY}

Abrasion and sore throat after a procedure requiring prolonged endotracheal intubation are common. Although this is certainly not life threatening, it is an annoyance for hours to days postoperatively. There are no studies explaining why some patients suffer with throat pain and others do not. One retrospective review of over 7500 cases identified several risk factors associated with postoperative sore throat. These include the female gender, age between 30 and 39, surgery within the oral cavity and around the neck, use of nitrous oxide for anesthesia, and duration of procedure greater than 2 hours. ${ }^{11}$ Although some of these risk factors are unavoidable, the study identifies a group of patients at higher risk who should be warned or told to expect some soreness postoperatively.

There is no standard treatment or approach that minimizes the degree of postoperative sore throat. Some use preoperative lidocaine spray or jelly to bathe the endotracheal tube. There is even a study comparing filling the tube cuff with $10 \mathrm{~mL}$ of saline versus $10 \%$ lidocaine. ${ }^{12}$ The researchers found that lidocaine in the endotracheal cuff decreased the incidence and 
lowered the severity of sore throat postoperatively. ${ }^{12}$ This is not widely accepted at this time, but it is an interesting area for further research. If appropriate for the patient and the procedure being performed, the laryngeal mask airway (LMA) can also be used to avoid sore throat.

\section{CONCLUDING THOUGHTS}

Safety and patient comfort in the operating room have become increasingly important. Preventable complications are always a concern to the surgeon, and the pressure to avoid complications during elective cosmetic procedures is greater now than ever before. Safe and effective patient positioning can avoid frustrating nerve damage and postoperative muscle and joint pain. Traditionally, surgeons have left patient positioning to the nursing and anesthesia staff. Because responsibility for postoperative complications due to poor patient positioning ultimately lies with the surgeon, he or she should take an active role and follow basic guidelines in patient positioning.

Attention to the head and neck during the procedure is imperative because of the risk of devastating retinal ischemia, brachial plexus injury, and damage to the vertebral vessels. Although eye injury during nonocular surgery is rare, it should be an avoidable complication. There are no strict guidelines for how to protect the cornea and globe from injury; however, it is commonly accepted that taping the eyes shut when possible helps to prevent injury. Further, protecting the eyes from excess pressure when in the prone position may help avoid the grave complication of retinal ischemia and blindness. Finally, inadvertent electrical burns in the operating room must be avoided by vigilant attention to equipment placement and correct usage of electrosurgical instruments.

Careful attention to patient safety must remain the number one goal of the surgeon. Consideration of the many patient safety issues allows a smooth perioperative course, thereby improving the cosmetic surgery experience while optimizing patient safety.

\section{REFERENCES}

1. Kohn LT, Corrigan JM, Donnaldson MS. To Err Is Human: Building a Safer Health System. National Institute of Medicine, Committee on Quality of Health Care in America. Washington, DC: National Academy Press; 1999

2. Vandam LD. Positioning of patients for operation. Stiefel $\mathrm{RH}$, Electricity, electrical safety, and instrumentation in the operating room. In: Rogers MC, ed. Principles and Practice of Anesthesiology. Baltimore, MD: Mosby Year Book; 1993: 703-745

3. Cucchiara RF, Faust RJ. Patient positioning. In: Miller RD, ed. Anesthesia. New York, NY: Churchill Livingstone; 1994: 1057-1073

4. Fritzlen T, Kremer M, Biddle C. The AANA Foundation Closed Malpractice Claims Study on nerve injuries during anesthesia care. AANA J 2003;71:347-352

5. Akinbingol G, Borman H, Maral T. Bilateral brachial plexus palsy after a prolonged surgical procedure of reduction mammoplasty, abdominoplasty, and liposuction. Ann Plast Surg 2002;49:219-220

6. Bund M, Heine J, Jaeger K. Complications due to patient positioning. Anesthesiological considerations. Anasthesiol Intensivmed Notfallmed Schmerzther 2005;40:329-339

7. Shermak M, Shoo B, Deune EG. Prone positioning precautions in plastic surgery. Plast Reconstr Surg 2006;117: 1584-1589

8. Roth S, Thisted RA, Erickson JP, et al. Eye injuries after nonocular surgery. A study of 60,965 anesthetics from 1988 to 1992. Anesthesiology 1996;85:1020-1027

9. Principles of Electrosurgery. Online. Valleylab 2006. Available at: http://www.valleylab.com/education/poes/index.html

10. Mann D. Reducing the hazard of burns and bovie pads. Plast Reconstr Surg 2000;106:947

11. Chen KT, Tzeng JI, Lu CL, et al. Risk factors associated with postoperative sore throat after tracheal intubation: an evaluation in the postanesthetic recovery room. Acta Anaesthesiol Taiwan. 2004;42:3-8

12. Altintas F, Bozkurt P, Kaya G, et al. Lidocaine $10 \%$ in the endotracheal tube cuff: blood concentrations, hemodynamic and clinical effects. Eur J Anaesthesiol 2000;17:436-442 